\title{
The significance of perioperative coagulation and fibrinolysis related parameters after lung surgery for predicting venous thromboembolism: a prospective, single center study
}

\author{
Bo Tian, Chunfeng Song, Hui Li, Wenqian Zhang, Qirui Chen, Shuo Chen, Yili Fu, Xiaoxing Hu, Bin \\ You, Tong Li, Bin Hu, Shengcai Hou \\ Department of Thoracic Surgery, Beijing Chao-Yang Hospital, Capital Medical University, Beijing 100020, China \\ Contributions: (I) Conception and design: B Tian, C Song, H Li; (II) Administrative support: H Li, B Hu, S Hou; (III) Provision of study materials \\ or patients: W Zhang, Q Chen, S Chen, Y Fu, X Hu, B You, T Li; (IV) Collection and assembly of data: B Tian, C Song; (V) Data analysis and \\ interpretation: B Tian; (VI) Manuscript writing: All authors; (VII) Final approval of manuscript: All authors. \\ Correspondence to: Hui Li. Department of Thoracic Surgery, Beijing Chao-Yang Hospital, Capital Medical University, Beijing 100020, China. \\ Email: huilee@vip.sina.com.
}

Background: The high incidence of venous thromboembolism (VTE) has been perceived in post thoracic surgery patients. However, the significance of perioperative coagulation and fibrinolysis related parameters after lung surgery for VTE predicting is not clear. To investigate that, we conducted a prospective single center study.

Methods: A total of 111 patients undergoing lung surgery were enrolled in this study, included 52 primary lung cancer patients and 59 benign lung disease patients from July 2016 to March 2017. Preoperative and postoperative days 1,3, and 5 coagulation and fibrinolysis related parameters were tested, including antithrombin (AT), fibrinogen degradation product (FDP), prothrombin time (PT), prothrombin time activity (PA), prothrombin time ratio (PR), international normalized ratio (INR), activated partial thromboplastin time (APTT), plasma fibrinogen (FBG), thrombin time (TT) and D-Dimer. The Doppler ultrasonography was performed before and after surgery for deep venous thrombosis (DVT) confirmation. Patients with new postoperative DVT, unexplained dyspnea, hemoptysis, chest pain, or high Caprini score $(\geq 9)$ were received further computer tomography pulmonary angiography (CTPA) for pulmonary embolism (PE). We used the area under receiver-operating-characteristic (ROC) curve to discriminate patients between those who developed VTE and those who did not. Single factor analysis was utilized to define risk factors associated with VTE.

Results: The overall incidence of VTE was $16.2 \%$ (18/111). The incidence of VTE in primary lung cancer patients was $23.1 \%(12 / 52)$, much higher than that in benign lung diseases $10.2 \%(6 / 59)$, but did not reach statistical significance $(\mathrm{P}=0.066)$. Among 18 VTE patients, $83.3 \%$ was DVT, $16.7 \%$ was DVT + PE and $72.2 \%$ was muscular veins of the calf thrombosis. D-Dimer was much higher in VTE group than that in non-VTE group preoperatively and at postoperative days $1,3(0.64 \pm 0.24$ vs. $0.33 \pm 0.06, \mathrm{P}=0.007 ; 3.14 \pm 0.75$ vs. $1.51 \pm 0.09, \mathrm{P}=0.005$, and $1.88 \pm 0.53$ vs. $0.76 \pm 0.05, \mathrm{P}=0.001$, respectively). And the ROC curve areas of preoperative and postoperative days 1, 3 of D-Dimer were 0.70, 0.71 and 0.74, respectively. And FDP was much higher in VTE group than that in non-VTE group at postoperative day $3(6.78 \pm 1.43$ vs. $3.79 \pm 0.15$, P=0.004). But AT, PT, PA, PR, INR, APTT, FBG and TT there were no significantly difference.

Conclusions: The overall incidence of VTE after lung surgery was $16.2 \%$. The patients with preoperative high D-Dimer should receive VTE prophylaxis.

Keywords: Lung surgery; venous thromboembolism (VTE); coagulation

Submitted Nov 13, 2017. Accepted for publication Mar 16, 2018.

doi: $10.21037 /$ jtd.2018.03.174

View this article at: http://dx.doi.org/10.21037/jtd.2018.03.174 


\section{Introduction}

Venous thromboembolism (VTE) is a common postoperative syndrome and a common cause of postoperative death, nevertheless it is also a preventable disease. VTE, which consisting pulmonary embolism (PE) and deep venous thrombosis (DVT), is a major cause of morbidity and mortality after the surgery. Especially the malignant tumor surgery characterizes a greater incidence of postoperative VTE. PE and DVT are same disease expressed differently by parts and stages. Virchow' triad including hypercoagulability, stasis and endothelial injury that contribute to the development of venous thrombosis. The surgery and tumor are the main causation of acquired hypercoagulability $(1,2)$. The overall incidence of VTE after operation is approximately $0.8 \%$ to $1.6 \%(3,4)$, whereas the incidence of VTE after lung cancer surgery is higher than that, from $0.2 \%$ to $19 \%$ (5). A previous study show that the mortality rate of patients with VTE increase 6-fold, and half of the postoperative deaths are caused by VTE, about 2/3 VTE events occur before discharge (6).

During the process of VTE formation, the activation of coagulation and fibrinolysis system is accompanied. Four phases are included in the typical clotting process, the initiation and formation of platelet plug; clotting process propagation by coagulation cascade; the termination of clotting by antithrombotic mechanisms; clot removal by fibrinolysis (7). The operation injures tissue directly, large number of tissue factor (TF) enters the blood in a short time and activate the exogenous coagulation system. Surgery also damages endothelial cells, exposes the elements such as subcutaneous collagen and so endogenous coagulation system could be activated. Therefore, exogenous coagulation starts firstly and then exogenous coagulation system activates endogenous coagulation system in order to maintain and complete blood coagulation process. At the same time fibrinolysis system is activated. However, the significance of perioperative coagulation and fibrinolysis related parameters after lung surgery for VTE predicting is not clear. To investigate that, we conduct a prospective single center study.

\section{Methods}

\section{Patients}

A single center, prospective study was performed with data collected from patients who had undergone lung resection at Beijing Chao-Yang Hospital from July 2016 to March 2017. The color Doppler ultrasonography was performed before and after operation for DVT confirmation. Patients with new postoperative DVT, unexplained dyspnea, hemoptysis, chest pain, or high Caprini score $(\geq 9)$, highly suspected $\mathrm{PE}$, further computer tomography pulmonary angiography (CTPA) examination was required. The VTE incidence was evaluated before discharge. Preoperative and postoperative days 1,3 , and 5 coagulation and fibrinolysis related parameters were test, including antithrombin (AT), fibrinogen degradation product (FDP), prothrombin time $(\mathrm{PT})$, prothrombin time activity $(\mathrm{PA})$, prothrombin time ratio (PR), international normalized ratio (INR), activated partial thromboplastin time (APTT), plasma fibrinogen (FBG), thrombin time (TT) and D-Dimer. We used the area under the receiver-operating-characteristic (ROC) curve to discriminate between patients between those who developed VTE and those who did not. Single factor analysis was constructed to define risk factors that associated with VTE. The institutional research ethics committee of the Beijing Chao-Yang Hospital approved the study, and all of the patients signed the informed consent preoperatively.

\section{Inclusion criteria}

The medical records of consecutive patients who underwent lung resection but without VTE prevention were reviewed. All the patients underwent open or video-assisted thoracic surgery (VATS) procedure.

\section{Exclusion criteria}

Patients received any perioperative prophylaxis or anticoagulation with heparin, warfarin, aspirin or lowmolecular-weight heparin; patients had been confirmed with DVT or PE before surgery; patients with missing information, especially those who did not undergo VTE related imaging examine, coagulation and fibrinolysis related parameters blood test before or after surgery.

\section{Statistical analysis}

Continuous variables were expressed as the mean \pm standard deviation (SD), or medians compared by analysis of Student's $t$-test or Wilcoxon test. Categorical variables were compared by either the $\chi^{2}$ test or Fisher's exact test. We used the area under the receiver-operating-characteristic (ROC) curve to discriminate patients between the patients who developed VTE and those who did not. In this study, the area under the ROC curve between 0.5 and 0.7 , between 0.7 
and 0.9 and $>0.9$ were considered low, moderate and high, respectively. Single factor analysis was constructed to define risk factors associated with VTE. All statistical analyses were carried out with SPSS 23.0 and SigmaPlot 12.0 for Windows. The null hypothesis was rejected for $\mathrm{P}<0.05$.

\section{Results}

From department of Thoracic Surgery, Beijing ChaoYang Hospital affiliated to Capital Medical University, 111 patients were enrolled in this study, included 52 primary lung cancer patients and 59 benign lung disease patients admitted for lung surgery from July 2016 to March 2017. Among those patients, there was no difference about sex, the mean age at the time of surgery was 62.8 years of VTE patients and 52.3 years of non-VTE patients $(\mathrm{P}=0.001)$. Postoperative VTE incidence was correlated with increasing ASA scores. The VTE incidence in the 1-2 and 3-5 groups was $12.9 \%$ and $50.0 \%$ respectively. The high-ASA patients group had significant higher incidence than the lowASA group ( $\mathrm{P}=0.010)$. BMI, postoperative hospital stay, operative time and intraoperative bleeding, of VTE patients were higher than those of non-VTE patients $(25.2 \pm 1.1 \mathrm{vs}$. $23.3 \pm 0.4, \mathrm{P}=0.058 ; 8.6 \pm 1.2$ vs. $7.2 \pm 0.3, \mathrm{P}=0.589 ; 189.1 \pm 15.5$ vs. $165.5 \pm 7.9, \mathrm{P}=0.183$, and $202.8 \pm 44.5$ vs. $176.2 \pm 17.9$, $\mathrm{P}=0.484$, respectively), but no significant differences were got. And we did not find that postoperative VTE was correlated with operative procedures, type of resection, pathology, lung cancer postoperative stages (Table 1). The overall VTE incidence was $16.2 \%$ (18 of 111). The VTE incidence after primary lung cancer surgery was $23.1 \%$ (12 of 52), which was higher than that of benign lung diseases $10.2 \%$ (6 of 59), but did not reach statistical significance $(\mathrm{P}=0.066)$. And VTE incidence of open surgery was higher than that of VATS surgery $25.0 \%$ vs. $13.8 \%(\mathrm{P}=0.314)$. When the complexity of the surgery increased from wedge/ segmentectomy to bilobectomy, the incidence increased from $14.3 \%$ to $33.3 \%(\mathrm{P}=0.684)$. Among $18 \mathrm{VTE}$ lung cancer patients, $83.3 \%$ was DVT [15] and $16.7 \%$ was DVT $+\mathrm{PE}[3] ; 13$ patients (72.2\%) manifested muscular veins of the calf thrombosis (Table 2).

Preoperative and postoperative days 1, 3 D-Dimer of VTE(+) patients was much higher than that of VTE(-) patients, which were $0.64 \pm 0.24$ vs. $0.33 \pm 0.06, \mathrm{P}=0.007$; $3.14 \pm 0.75$ vs. $1.51 \pm 0.09, \mathrm{P}=0.005$ and $1.88 \pm 0.53$ vs. $0.76 \pm 0.05, \mathrm{P}=0.001$, respectively. But at day $5, \mathrm{D}$-Dimer has no statistical difference between $\mathrm{VTE}(+)$ patients and VTE(-) patients $(2.53 \pm 0.38$ vs. $1.88 \pm 0.12, \mathrm{P}=0.099)$. And postoperative days $3 \mathrm{FDP}$ of $\mathrm{VTE}(+)$ patients was much higher than that of VTE(-) patients, which was $6.78 \pm 1.43$ vs. $3.79 \pm 0.15, \mathrm{P}=0.004$. But for other parameters, there were no significantly difference (Table 3). And the ROC curve areas of preoperative and postoperative days 1, 3, 5 of D-Dimer were $0.70,0.71,0.74$ and 0.62 , respectively (Figure 1). When we took Youden index as the cutoff point, Pre-D-Dimer Youden index $>0.15$, the sensitivity was $94.4 \%$, specificity was $44.1 \%(\mathrm{P}=0.007)$, D1-D-Dimer Youden index $>1.26$, the sensitivity was $83.3 \%$, specificity was $47.3 \%(P=0.005)$, D3-D-Dimer Youden index $>0.95$, the sensitivity was $66.7 \%$, specificity was $74.2 \%(\mathrm{P}=0.001)$, D5-D-Dimer Youden index $>3.44$, the sensitivity was $33.3 \%$, specificity was $92.5 \%(\mathrm{P}=0.099)$ (Table 4).

\section{Discussion}

The high VTE incidence has been perceived in post-surgery patients. VTE is the most common complication and the main cause of death in the thoracic surgery after operation. This study finds out that the overall VTE incidence is $16.2 \%$. The VTE incidence after primary lung cancer surgery is $23.1 \%$, which is much higher than that of benign lung diseases (10.2\%). This finding is consistent with recent study (5). Lung cancer may be responsible for the higher incidence of thrombotic events associated with cancer, but do not reach statistical significance. Among those 111 patients, the median age at the time of surgery was 62.8 years old for the VTE patients and 52.3 years old for the nonVTE patients. VTE is a predominant disease for older ages (8). Postoperative VTE incidence is correlated with increasing ASA scores. The VTE incidence in the 1-2 and 3-5 groups was $12.9 \%$ and $50.0 \%$ respectively. The highASA patients group had significant higher incidence than the low-ASA group. Older and high ASA patients are seems more likely to have VTE after operation. In this study, we found that postoperative VTE incidence did not correlate with lung cancer pathology and postoperative stages, while previous literature showed that the VTE incidence of adenocarcinoma histology was 2 -fold of squamous cell carcinoma (9). VTE is more common in late-stage cancer patients, and its incidence increases with the tumor progression after operation (10). The product of TF and mucin by adenocarcinomas may be related to this higher risk (11). And also we find open surgery VTE incidence is higher than VATS surgery, which are $25.0 \%$ and $13.8 \%$ respectively. In addition, the VTE incidence of wedge/ segmentectomy and bilobectomy operation are $14.3 \%$ and 
Table 1 General and operation information for patients

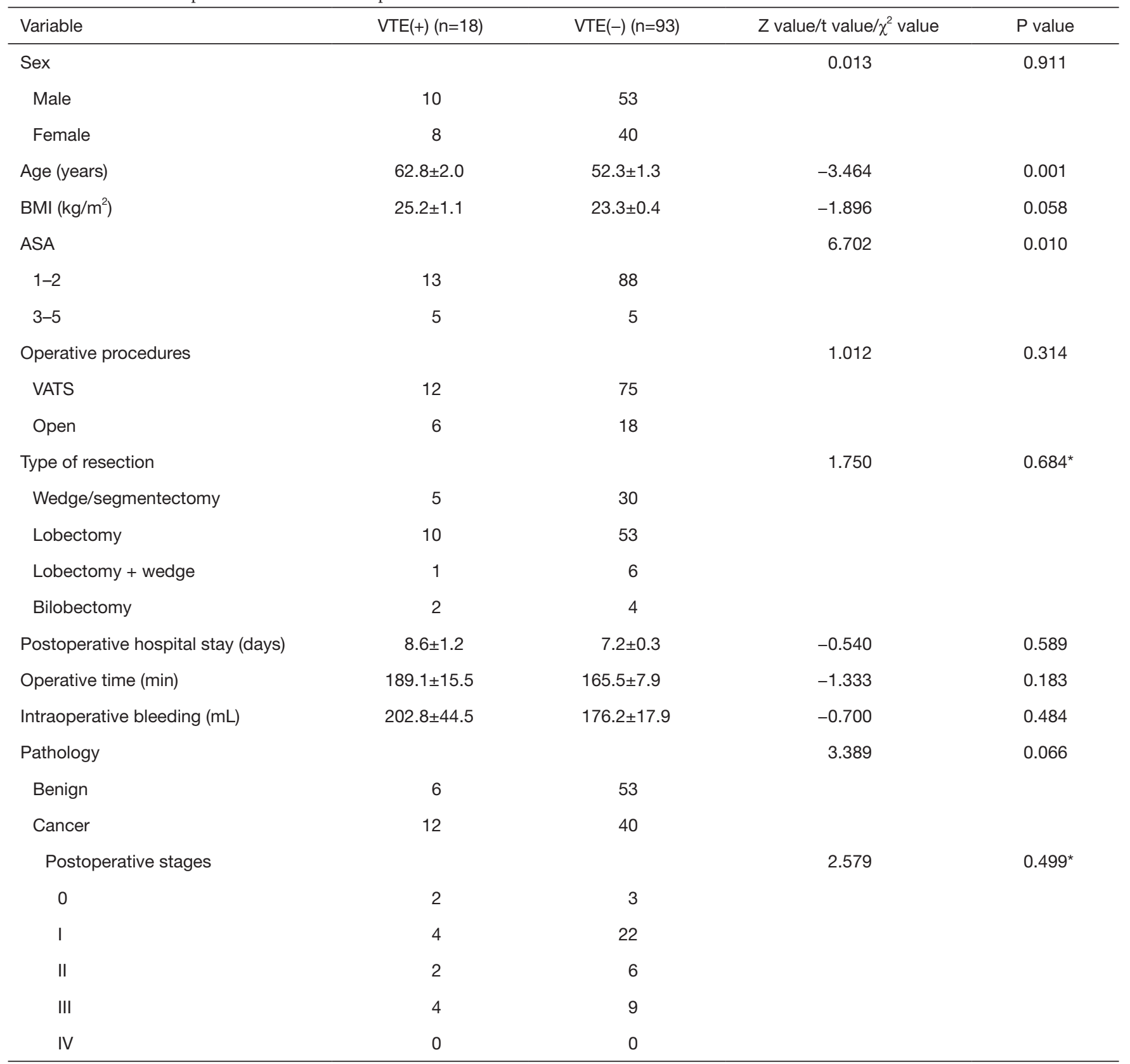

*, Fisher's exact test. VTE, venous thromboembolism; BMI, body mass index; VATS, video-assisted thoracoscopic surgery.

$33.3 \%$ respectively, which means that when the complexity of the surgery increases, the VTE incidence is elevated too. And BMI, postoperative hospital stay, operative time and intraoperative bleeding of VTE patients are higher than those of non-VTE patients. A literature demonstrated that prevalence of VTE is $8.9 \%$ after total pneumonectomy (12). VTE incidence is higher than we thought previously, the different time of examination could be one reason which caused this different outcome. In some researches, patients received Ultrasound examination for lower limb venous only when they complained with lower limb swelling, pain and so on. However, in our study, Ultrasound examination was routinely performed before and after the surgery in order to get an accurate and timely diagnosis with VTE. 
Table 2 VTE information for patients

\begin{tabular}{lcc}
\hline Variable & Cases $(n=18)$ & Ratio \\
\hline $\mathrm{DVT}(+) \mathrm{PE}(-)$ & 15 & 83.3 \\
$\mathrm{DVT}(-) \mathrm{PE}(+)$ & 0 & 0 \\
$\mathrm{DVT}(+) \mathrm{PE}(+)$ & 3 & 16.7 \\
\hline
\end{tabular}

VTE, venous thromboembolism; DVT, deep venous thrombosis; $\mathrm{PE}$, pulmonary embolism.

Table 3 Perioperative coagulation and fibrinolysis related parameters results

\begin{tabular}{|c|c|c|c|}
\hline Variable & $\begin{array}{l}\operatorname{VTE}(+) \\
(n=18)\end{array}$ & $\begin{array}{l}\operatorname{VTE}(-) \\
(n=93)\end{array}$ & $\begin{array}{c}Z \text { value/t } \\
\text { value } / \chi^{2} \text { value }\end{array}$ \\
\hline
\end{tabular}

\begin{tabular}{lllll}
\hline AT (\%) & & & & \\
Pre-op & $89.73 \pm 3.19$ & $91.18 \pm 1.36$ & -0.952 & 0.341 \\
D1 & $81.31 \pm 3.46$ & $81.84 \pm 1.16$ & -0.260 & 0.795 \\
D3 & $78.39 \pm 2.98$ & $83.02 \pm 1.18$ & -1.545 & 0.125 \\
D5 & $82.03 \pm 3.10$ & $86.50 \pm 1.12$ & -1.545 & 0.125
\end{tabular}

$\operatorname{FDP}(\mu \mathrm{g} / \mathrm{mL})$

$\begin{array}{lrrrr}\text { Pre-op } & 2.21 \pm 0.63 & 1.96 \pm 0.29 & -1.053 & 0.292 \\ \text { D1 } & 10.68 \pm 3.14 & 6.04 \pm 0.41 & -1.553 & 0.121 \\ \text { D3 } & 6.78 \pm 1.43 & 3.79 \pm 0.15 & -2.878 & 0.004 \\ \text { D5 } & 7.46 \pm 1.13 & 5.56 \pm 0.26 & -1.476 & 0.140 \\ \text { PT (s) } & & & & \\ \text { Pre-op } & 12.10 \pm 0.25 & 12.15 \pm 0.08 & -1.137 & 0.255 \\ \text { D1 } & 12.48 \pm 0.30 & 12.55 \pm 0.11 & -0.344 & 0.731 \\ \text { D3 } & 12.29 \pm 0.18 & 12.47 \pm 0.12 & -0.536 & 0.592 \\ \text { D5 } & 12.43 \pm 0.12 & 12.62 \pm 0.07 & -0.974 & 0.330\end{array}$

PA (\%)

$\begin{array}{lllll}\text { Pre-op } & 90.30 \pm 2.05 & 90.32 \pm 0.74 & -0.468 & 0.639 \\ \text { D1 } & 84.19 \pm 2.07 & 84.49 \pm 0.88 & -0.138 & 0.891 \\ \text { D3 } & 87.37 \pm 1.71 & 86.39 \pm 0.85 & -0.564 & 0.573 \\ \text { D5 } & 86.95 \pm 1.02 & 85.06 \pm 0.63 & -1.209 & 0.227\end{array}$

PR

\begin{tabular}{lllll} 
Pre-op & $1.04 \pm 0.02$ & $1.04 \pm 0.01$ & -0.818 & 0.414 \\
D1 & $1.10 \pm 0.02$ & $1.09 \pm 0.01$ & 0.164 & 0.870 \\
D3 & $1.07 \pm 0.02$ & $1.08 \pm 0.01$ & -0.273 & 0.785 \\
D5 & $1.08 \pm 0.01$ & $1.09 \pm 0.01$ & -0.946 & 0.344 \\
\hline
\end{tabular}

Table 3 (continued)
Table 3 (continued)

\begin{tabular}{ccccc}
\hline Variable & $\begin{array}{c}\text { VTE(+) } \\
(n=18)\end{array}$ & $\begin{array}{c}\text { VTE(-) } \\
(n=93)\end{array}$ & $\begin{array}{c}Z \text { value/t } \\
\text { value/ } \chi^{2} \text { value }\end{array}$ & P value \\
\hline INR & & & & \\
Pre-op & $1.02 \pm 0.02$ & $1.02 \pm 0.01$ & -0.493 & 0.622 \\
D1 & $1.09 \pm 0.02$ & $1.08 \pm 0.01$ & -0.008 & 0.994 \\
D3 & $1.05 \pm 0.02$ & $1.06 \pm 0.01$ & -0.493 & 0.622 \\
D5 & $1.05 \pm 0.01$ & $1.07 \pm 0.01$ & -1.191 & 0.234
\end{tabular}

APTT (s)

$\begin{array}{lllll}\text { Pre-op } & 28.60 \pm 1.00 & 29.91 \pm 0.46 & -0.920 & 0.357 \\ \text { D1 } & 30.91 \pm 1.39 & 31.45 \pm 0.52 & -0.176 & 0.860 \\ \text { D3 } & 33.47 \pm 1.38 & 32.80 \pm 0.56 & -0.788 & 0.431 \\ \text { D5 } & 30.51 \pm 1.11 & 31.49 \pm 0.43 & -0.894 & 0.373\end{array}$

$\mathrm{FBG}(\mathrm{mg} / \mathrm{dL})$

$\begin{array}{lrrrr}\text { Pre-op } & 308.44 \pm 29.08 & 271.79 \pm 7.66 & -0.736 & 0.462 \\ \text { D1 } & 323.19 \pm 26.45 & 297.15 \pm 7.76 & -0.564 & 0.573 \\ \text { D3 } & 520.07 \pm 23.77 & 488.32 \pm 10.68 & -1.312 & 0.189 \\ \text { D5 } & 526.44 \pm 23.69 & 498.85 \pm 12.49 & -1.040 & 0.298 \\ \text { TT (s) } & & & & \\ \text { Pre-op } & 18.00 \pm 0.18 & 18.15 \pm 0.10 & -0.348 & 0.728 \\ \text { D1 } & 16.59 \pm 0.18 & 16.71 \pm 0.10 & -0.364 & 0.715 \\ \text { D3 } & 16.02 \pm 0.18 & 15.88 \pm 0.10 & -1.310 & 0.190 \\ \text { D5 } & 16.11 \pm 0.11 & 16.08 \pm 0.08 & -0.866 & 0.387\end{array}$

D-Dimer (mg/L)

\begin{tabular}{lllll} 
Pre-op & $0.64 \pm 0.24$ & $0.33 \pm 0.06$ & -2.700 & 0.007 \\
D1 & $3.14 \pm 0.75$ & $1.51 \pm 0.09$ & -2.820 & 0.005 \\
D3 & $1.88 \pm 0.53$ & $0.76 \pm 0.05$ & -3.268 & 0.001 \\
D5 & $2.53 \pm 0.38$ & $1.88 \pm 0.12$ & -1.648 & 0.099 \\
\hline
\end{tabular}

VTE, venous thromboembolism; AT, antithrombin; FDP, fibrinogen degradation product; $\mathrm{PT}$, prothrombin time; PA, prothrombin time activity; PR, prothrombin time ratio; INR, international normalized ratio; APTT, activated partial thromboplastin time; FBG, plasma fibrinogen; TT, thrombin time.

Because VTE usually develops before significant clinical manifestation occurs, we showed the VTE incidence in postoperative patients is higher than that in other studies, indicating that the real incidence may be underestimated previously.

Among 18 VTE patients, VTE mainly happens in deep 
vein thrombosis, with an occupation of $83.3 \%$ over total population, while pure $\mathrm{PE}$ is less likely to happen, with an incidence rate of $0 \%$. It is reported that $\mathrm{PE}$ was confirmed in $15.2 \%$ of postoperative deaths by autopsy, indicating that it is a frequently fatal postoperative complication (13). Among those 18 patients, 13 (72.2\%) of which manifested distal isolated calf muscular venous thrombosis. It is generally acknowledged that the calf muscular venous thrombosis has a lower venous extend risk than axial venous thrombosis (such as fibular venous and femoral venous thrombosis) (14). Whether to apply anticoagulation therapy for calf muscular venous thrombosis is still controversial, presently in our department we have taken standardize anticoagulation therapy, and closely follow-up with post operational visit, the therapeutic effect and risks still remain to be observed.

A literature demonstrated that the fatal recurrence rate of

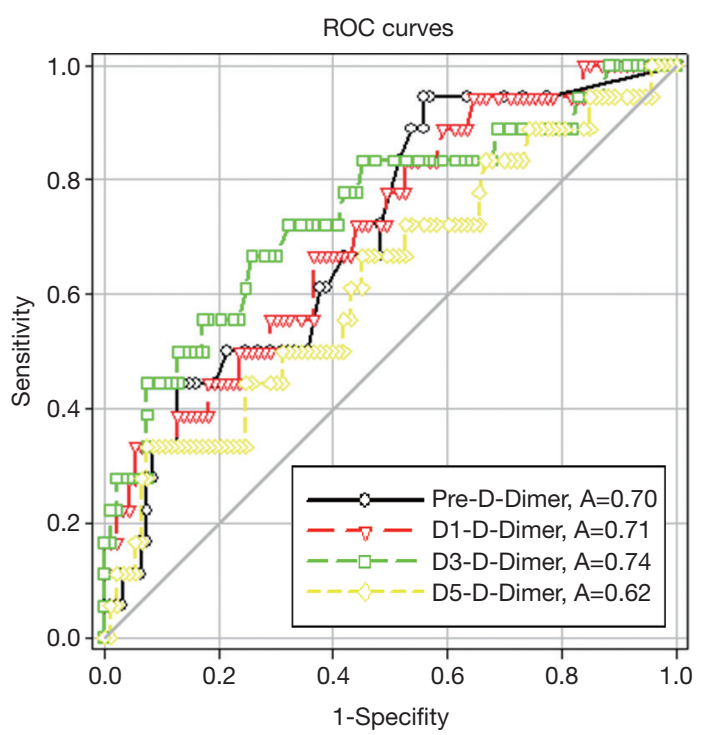

Figure 1 ROC curves of preoperative and postoperative days 1, 3 and 5 of D-Dimer. ROC, receiver-operating-characteristic.
VTE is as high as $5 \%$ to $9 \%$ in patients, yet the occurrence of postoperative VTE is occult, and lack of characteristic clinical manifestations (15). It is mainly diagnosed by lower limb venous color Doppler ultrasonography and the CT imaging of pulmonary blood vessels. Once DVT patients form PE, it will cause serious damage to the patient's health, even lead to sudden death. Malignant tumor patients with VTE have a poorer prognosis, the average survival period is much shorter. Perioperative VTE is the second most common complication for inpatients and the third most common cause of mortality, leading to the delay of postoperative recovery, increase treatment costs and longer hospital stays. This will further induce a great adverse effect on the prognosis of surgical patients and become one of the most important causes of unanticipated death after surgery (16), so the prevention of VTE is very important.

Although a previous meta-analysis (17) stated that the incidence of DVT patients with pharmacological thromboprophylaxis was relatively decreased compared with those without, the incidence of bleeding events also has been found out experienced a significant increase. A significant difference has been detected at the time of the first dose usage preoperatively, but no clue on postoperative patients. A literature (18) demonstrated that it is safe and feasible to take preoperative use of heparin for thromboprophylaxis in Chinese patients. However, due to the usage risks of heparin, the timing of anticoagulation is important.

In our study, preoperative and postoperative days 1, 3 D-Dimer of $\operatorname{VTE}(+)$ patients is much higher than that of $\operatorname{VTE}(-)$ patients, which are $0.64 \pm 0.24$ vs. $0.33 \pm 0.06$, $\mathrm{P}=0.007 ; 3.14 \pm 0.75$ vs. $1.51 \pm 0.09, \mathrm{P}=0.005$ and $1.88 \pm 0.53$ vs. $0.76 \pm 0.05, \mathrm{P}=0.001$, respectively. And postoperative days $3 \mathrm{FDP}$ of $\operatorname{VTE}(+)$ patients is much higher than that of VTE(-) patients, which is $6.78 \pm 1.43$ vs. $3.79 \pm 0.15, \mathrm{P}=0.004$. Patients with VTE positive have shown a significant increase of D-Dimer before surgery, almost 2-fold higher, suggesting that such patients show a preoperative state

Table 4 Perioperative ROC curve area and Youden index and corresponding sensitivity and specificity of D-Dimer

\begin{tabular}{|c|c|c|c|c|c|c|}
\hline Variable & ROC curve area & $95 \%$ confidence interval & Youden index & Sensitivity (\%) & Specificity (\%) & $P$ value \\
\hline D1-D-Dimer & 0.7106 & $0.5809-0.8402$ & $>1.26$ & 83.3 & 47.3 & 0.005 \\
\hline D3-D-Dimer & 0.7440 & $0.6054-0.8827$ & $>0.95$ & 66.7 & 74.2 & 0.001 \\
\hline D5-D-Dimer & 0.6231 & $0.4729-0.7732$ & $>3.44$ & 33.3 & 92.5 & 0.099 \\
\hline
\end{tabular}

ROC, receiver-operating-characteristic. 
of thrombosis. And the postoperative D-Dimer is rapidly elevated, with significant statistical differences between the first and third day after surgery. D-Dimer is cross linked fibrin fibrinolysis enzyme hydrolysis to produce a specific molecular markers, and its level reflects the extent of the secondary fibrinolysis inside body, can be used as high coagulation state in the body and an important index of hyper fibrinolysis.

And the ROC curve areas of preoperative and postoperative days $1,3,5$ of D-Dimer are $0.70,0.71,0.74$ and 0.62 , respectively. When take Youden index as the cutoff point, D3-D-Dimer Youden index $>0.95$, the sensitivity is $66.7 \%$, specificity is $74.2 \%$. From the ROC curve area preoperative and postoperative days 1, $3 \mathrm{D}$-Dimer have a moderate discrimination between patients who develop VTE and those who do not. And D3-D-Dimer has the highest ROC curve area, and corresponding sensitivity and specificity is both high. So we think the patients with preoperative high D-Dimer should receive VTE prophylaxis. But further research is still needed to confirm it.

The main objective of the treatment of deep vein thrombosis is to prevent $\mathrm{PE}$, especially in the early stage of the course of the disease, since the thrombus and the wall of blood vessels are not tight and easy to fall off. In order to avoid the serious threat of $\mathrm{PE}$, all high-risk patients with DVT, such as the patients with femoral head fracture, larger orthopedic or pelvic surgery and the elderly with high blood viscosity should be prevented in advance. The ninth edition antithrombotic and thrombolysis therapy evidence-based guidelines were published in 2012 by American thoracic society (American College of Chest Physicians, ACCP) (19), suggesting VTE therapy and prevention for orthopedic surgery patients. But there are no specific guidelines about VTE therapy and prevention after thoracic surgery. Actually, in China, prophylaxis is not the routine management in most hospitals after thoracic surgery. The main reason is majority of thoracic surgeons in China does not realize the high incidence of postoperative VTE. Secondly, there are no relevant data from China to support prophylaxis after thoracic surgery. Through the early questionnaires survey researched on 1,150 thoracic surgery doctors found that $46.9 \%$ of thoracic surgeons did not prevent against VTE, $56.2 \%$ of them think that no thoracic surgery related guidelines is the main reason that they did not take the prevention and $99.6 \%$ of those doctors considered that VTE prevention was quite necessary. Therefore, through this research, we find out a relatively accurate data on the incidence of postoperative VTE after lung surgery, but this is just a single center study, in order to indicate the most meaningful risk factors that could explain the prevalence of postoperative VTE, set the most suitable risk score standard for Chinese patients, and eventually develop the specialists consensus and guidelines on VTE prevention for Chinese thoracic surgery, there are many works need to do.

\section{Acknowledgements}

Thank you for my wife Fuli Liu, for taking care of our child and family while I am studying for my doctorate.

\section{Footnote}

Conflicts of Interest: The authors have no conflicts of interest to declare.

Ethical Statement: This study was approved by the Institutional Research Ethics Committee of Beijing Chao-Yang Hospital, Capital Medical University (ID: 2017-Ke-1). All the patients signed the informed consent preoperatively. The study outcomes will not affect the future management of the patients.

\section{References}

1. Bagot CN, Arya R. Virchow and his triad: a question of attribution. Br J Haematol 2008;143:180-90.

2. Goldhaber SZ. Risk factors for venous thromboembolism. J Am Coll Cardiol 2010;56:1-7.

3. Nelson RE, Grosse SD, Waitzman NJ, et al. Using multiple sources of data for surveillance of postoperative venous thromboembolism among surgical patients treated in Department of Veterans Affairs hospitals, 2005-2010. Thromb Res 2015;135:636-42.

4. Merkow RP, Bilimoria KY, McCarter MD, et al. Postdischarge venous thromboembolism after cancer surgery: extending the case for extended prophylaxis. Ann Surg 2011;254:131-7.

5. Christensen TD, Vad H, Pedersen S, et al. Venous thromboembolism in patients undergoing operations for lung cancer: a systematic review. Ann Thorac Surg 2014;97:394-400.

6. Agnelli G, Bolis G, Capussotti L, et al. A clinical outcomebased prospective study on venous thromboembolism after cancer surgery: the @RISTOS project. Ann Surg 2006;243:89-95. 
7. Muciño-Bermejo J, Carrillo-Esper R, Uribe M, et al. Coagulation abnormalities in the cirrhotic patient. Ann Hepatol 2013;12:713-24.

8. Cushman M, Tsai AW, White RH, et al. Deep vein thrombosis and pulmonary embolism in two cohorts: the longitudinal investigation of thromboembolism etiology. Am J Med 2004;117:19-25.

9. Chew HK, Davies AM, Wun T, et al. The incidence of venous thromboembolism among patients with primary lung cancer. J Thromb Haemost 2008;6:601-8.

10. Yang Y, Zhou Z, Niu XM, et al. Clinical analysis of postoperative venous thromboembolism risk factors in lung cancer patients. J Surg Oncol 2012;106:736-41.

11. Corrales-Rodriguez L, Blais N. Lung cancer associated venous thromboembolic disease: a comprehensive review. Lung Cancer 2012;75:1-8.

12. Raja S, Idrees JJ, Blackstone EH, et al. Routine venous thromboembolism screening after pneumonectomy: The more you look, the more you see. J Thorac Cardiovasc Surg 2016;152:524-32 e2.

13. Daddi G, Milillo G, Lupattelli L, et al. Postoperative pulmonary embolism detected with multislice computed tomography in lung surgery for cancer. J Thorac Cardiovasc Surg 2006;132:197-8.

Cite this article as: Tian B, Song C, Li H, Zhang W, Chen Q, Chen S, Fu Y, Hu X, You B, Li T, Hu B, Hou S. The significance of perioperative coagulation and fibrinolysis related parameters after lung surgery for predicting venous thromboembolism: a prospective, single center study. J Thorac Dis 2018;10(4):2223-2230. doi: 10.21037/jtd.2018.03.174
14. Kearon C, Akl EA, Ornelas J, et al. Antithrombotic Therapy for VTE Disease: CHEST Guideline and Expert Panel Report. Chest 2016;149:315-52.

15. Douketis JD, Gu CS, Schulman S, et al. The risk for fatal pulmonary embolism after discontinuing anticoagulant therapy for venous thromboembolism. Ann Intern Med 2007;147:766-74.

16. Heit JA. The epidemiology of venous thromboembolism in the community. Arterioscler Thromb Vasc Biol 2008;28:370-2.

17. Guo Q, Huang B, Zhao J, et al. Perioperative Pharmacological Thromboprophylaxis in Patients With Cancer: A Systematic Review and Meta-analysis. Ann Surg 2017;265:1087-93.

18. Deng HY, Shi CL, Li G, et al. The safety profile of preoperative administration of heparin for thromboprophylaxis in Chinese patients intended for thoracoscopic major thoracic surgery: a pilot randomized controlled study. J Thorac Dis 2017;9:1065-72.

19. Gould MK, Garcia DA, Wren SM, et al. Prevention of VTE in nonorthopedic surgical patients: Antithrombotic Therapy and Prevention of Thrombosis, 9th ed: American College of Chest Physicians Evidence-Based Clinical Practice Guidelines. Chest 2012;141:e227S-e277S. 